\title{
NOTAS DE COMPORTAMIENTO DEFENSIVO DE DOS SALAMANDRAS DE LA REGIÓN DE TALAMANCA, COSTA RICA
}

\section{NOTES ON DEFENSIVE BEHAVIOR OF TWO SALAMANDERS OF TALAMANCA REGION, COSTA RICA}

\author{
MARIA José CAMACHo-DuRáN ${ }^{1 *}$ \& Diego A. GómeZ-Hoyos ${ }^{2}$ \\ ${ }^{1}$ Semillero de Ecología y Conservación de Anfibios y Reptiles, Departamento de Ecología y Territorio, Pontificia Universidad Javeriana, \\ Bogotá, Colombia. \\ ${ }^{2}$ ProCAT Colombia/Internacional \& Sierra to Sea Institute Costa Rica, Las Alturas, Puntarenas, Costa Rica \\ *Correspondencia: camacho_m@javeriana.edu.co
}

Abstract.- Salamanders have a variety of defensive and antipredator behavioral strategies. In this report, we describe the defensive behavior of a Bolitoglossa gomezi and Oedipina savagei individual at Las Tablas Protected Zone, Costa Rica. The behavior of the B. gomezi consisted of flipping its body to the ground and remaining immobile; the O. savagei individual held its tail elevated at a $90^{\circ}$ angle with its body. The strategy of B. gomezi has been related to predator difficulty in relocating the prey, and the strategy of O. savagei with attracting predators to the tail, the most dispensable part of its body. Defensive behavior in salamanders is frequent but reports of field observations are scarce in the literature. We encourage herpetologists to fill this knowledge gap.

Keywords.- Antipredator, Bolitoglossa, Caudata, natural history, Oedipina, thanatosis.

Resumen.- Las salamandras tienen una variedad de estrategias comportamentales de defensa o antidepredatorias. En este reporte describimos los comportamientos defensivos de un individuo de Bolitoglossa gomezi y Oedipina savagei en la Zona Protectora Las Tablas, Costa Rica. El comportamiento de B. gomezi consistió en lanzarse a la hojarasca y quedarse inmóvil; el individuo de O. savagei levantó su cola cerca de $90^{\circ}$ con relación a su cuerpo. La estrategia de B. gomezi se ha relacionado con dificultad de los depredadores para relocalizar la presa, y la estrategia de O. savagei con atraer a los depredadores a la cola, una parte del cuerpo menos comprometedora. Los comportamientos defensivos en las salamandras son frecuentes, pero los reportes de observaciones en campo son escasos en la literatura. Animamos a los herpetólogos a llenar estos vacíos de conocimiento.

Palabras clave.- Antidepredatorio, Bolitoglossa, Caudata, historia natural, Oedipina, tanatosis.

Costa Rica alberga 53 especies reconocidas de salamandras (Zumbado-Hulate et al., 2019) y su mayor diversidad se encuentra en la Cordillera de Talamanca, especialmente en la frontera con Panamá (Dodd \& Bodrie, 1976; Wake et al., 2007; Bolaños \& Wake, 2009). En esta región los estudios de este grupo de anfibios han estado dedicados principalmente a su sistemática y taxonomía (Boza-Oviedo et al., 2012; Wake et al., 2007; Kubicki \& Arias, 2016). Estos esfuerzos han sido importantes, porque la delimitación de las especies ha permito conocer la diversidad de salamandras en el área. Sin embargo, se deben realizar esfuerzos paralelos que busquen el estudio y reporte de información en ecología, biología e historia natural de las salamandras con el fín de llenar los vacíos de conocimiento que existen en este grupo.

Además de su diversidad taxonómica, las salamandras terrestres también exponen diversidad de comportamientos (Brodie, 1977). Por ejemplo, los mecanismos de defensa de las salamandras han convergido en diversas estrategias antidepredatorias que pueden ser morfológicas (coloración aposemática, camuflaje y autotomía caudal), comportamentales (posiciones del cuerpo o partes del cuerpo, movimientos e inmovilidad), y fisiológicas (secreción de sustancias tóxicas y nocivas) (Brodie, 1977; Dodd \& Bodrie, 1976).

Algunos estudios en la región Neotropical han descrito los comportamientos antidepredatorios o de defensa de salamandras. Para algunas especies del género Bolitoglossa se han descrito estrategias comportamentales y fisiológicas ante la presencia o ataque de serpientes (Brodie et al., 1991; Ducey \& Brodie, 1991; Ducey et al., 1993). También se ha reportado el efecto de condiciones específicas de temperatura en comportamientos de inmovilidad (Dodd \& Brodie, 1976) y respuestas de autotomía caudal ante pérdida de agua (Vial, 1968). Estos comportamientos de defensa que exponen las salamandras son poco estudiados 
o reportados en la actualidad en esta región. Por lo tanto, en esta contribución reportamos los comportamientos defensivos observados en Bolitoglossa gomezi y Oedipina savagei en la Zona Protectora Las Tablas y su zona de amortiguamiento en Costa Rica.

La Zona Protectora Las Tablas y su zona de amortiguamiento se encuentran en el cantón de Cotobrus, provincia de Puntarenas-Costa Rica (Fig. 1). Realizamos los registros los días 18 de mayo de 2019 durante encuentros casuales para B. gomezi, así como el 19 de junio de 2019 para O. savagei en un transecto lineal utilizado durante una evaluación del efecto de borde sobre la herpetofauna. Los reportes corresponden a las ubicaciones geográficas $8.95725^{\circ} \mathrm{N}$ y $82.82906^{\circ} \mathrm{W}$ ca. $1600 \mathrm{~m}$. s. n. m. para B. gomezi, así como $8.90426^{\circ} \mathrm{N}$ y $82.85826^{\circ} \mathrm{W} 1200 \mathrm{~m}$. s. n. m., para O. savagei (Fig. 1). El registro de B. gomezi corresponde a un individuo que encontramos sobre una planta a $50 \mathrm{~cm}$ del suelo (Fig. 2A) a las 22:33 horas, en un área de bosque maduro en el Cerro Chai. Por otra parte, realizamos el registro de O. savagei en un ecotono de bosque-pastizal, sobre una planta a $20 \mathrm{~cm}$ del suelo (Fig. 3A) a las 23:04 horas.

Identificamos el individuo como B. gomezi con base en los caracteres que proponen Wake et al. (2007) en la descripción original de la especie, para distinguirla de otras especies de salamandras de la zona: cabeza levemente más ancha que el cuello y menos distintiva del tronco; la palmeadura de las patas, especialmente la extensión de la membrana entre los dedos I y II, la cual es menos extensa que en B. bramei (la especie más parecida); puntos y manchas blancas ventrales, especialmente grandes debajo de la cola (Fig. 2B). Por su parte, el individuo de O. savagei lo identificamos y diferenciamos de especies similares como O. alleni basados en los caracteres de su descripción original (García-París \& Wake, 2000): raya clara dorsal (Fig. 3B), ausente en O. alleni; el dedo más largo del pie termina en punta, más redondeado en O. alleni. Además, O. savagei ocurre en mayores elevaciones respecto a O. alleni (García-París \& Wake, 2000). Sin embargo, aclaramos que los individuos encontrados no fueron colectados, por lo que existe incertidumbre en su identificación.

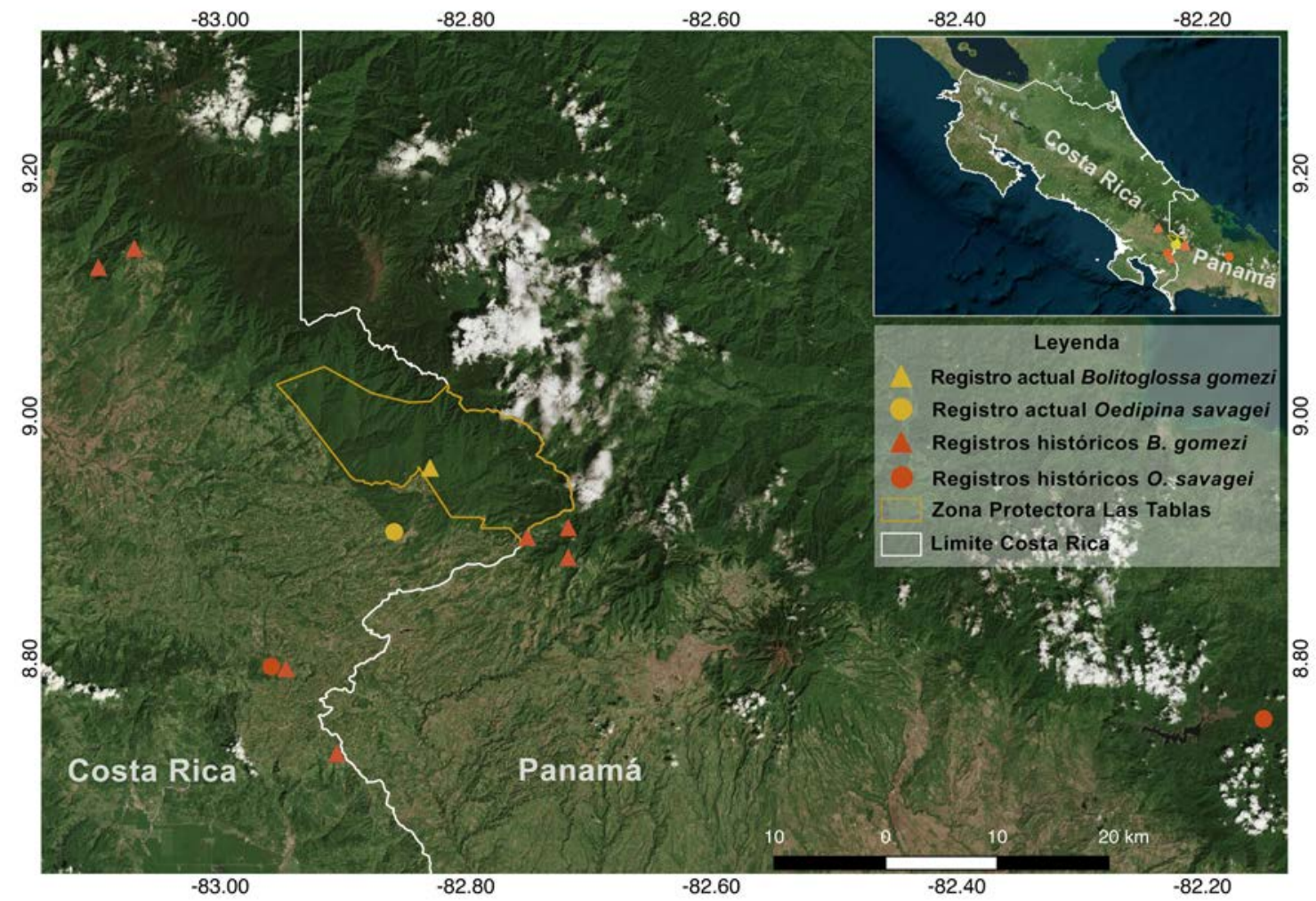

Figure 1. Historic and current records of Bolitoglossa gomezi and Oedipina savagei at Las Tablas Protected Zone and its buffer zone, Costa Rica. Historic records were taken from: Boza-Oviedo et al., 2012; García-París y Wake, 2000; Wake et al., 2007.

Figura 1. Registros históricos y actuales de Bolitoglossa gomezi y Oedipina savagei en la Zona Protectora Las Tablas y su zona de amortiguamiento, Costa Rica. Registros históricos tomados de: Boza-Oviedo et al., 2012; García-París y Wake, 2000; Wake et al., 2007. 


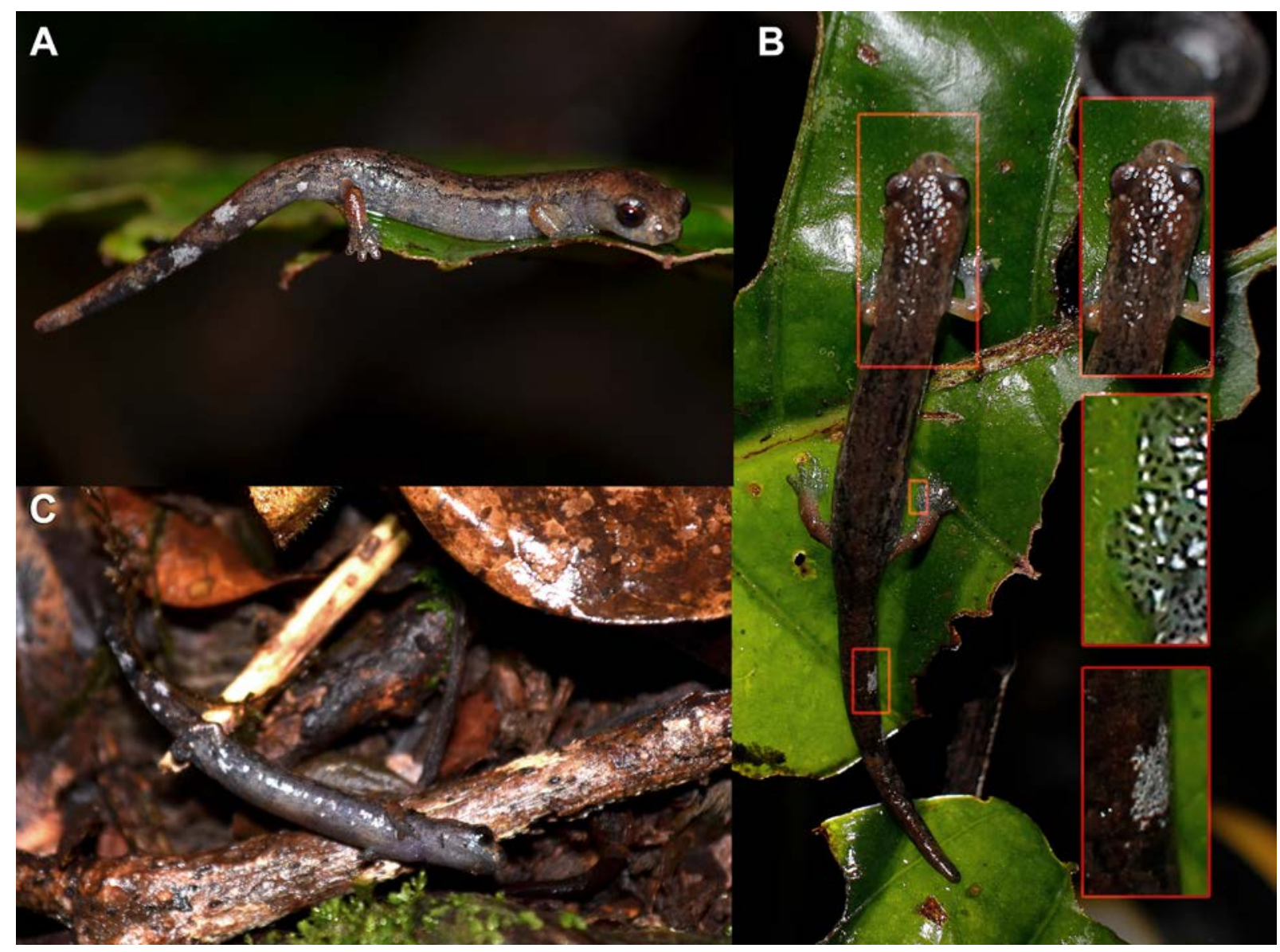

Figure 2. Bolitoglossa gomezi individual found at Cerro Chai, Las Tablas Protected Zone, Costa Rica (A). Characteristics used for identification: head slightly wider than neck, but less distinct from the trunk, digital webbing in toes I and II less extent than in B. bramei, light spots and blotches in venter, especially larger on the underside of the tail (B); behavior mechanism known as thanatosis (C).

Figura 2. Individuo de Bolitoglossa gomezi encontrado en el Cerro Chai, Zona Protectora Las Tablas, Costa Rica (A). Características utilizadas para su identificación: cabeza levemente más ancha que el cuello, menos distintiva del tronco, membrana entre dedos I y II menos extensa que en B. bramei, puntos y manchas blancas ventrales especialmente grandes en la cola (B); comportamiento de defensa conocido como tanatosis (C).

Ambas salamandras encontradas mostraron comportamientos defensivos. El individuo de B. gomezi al ser manipulado se lanzó a la hojarasca, tomó una posición ventrolateraly se quedó inmóvil en un comportamiento conocido como tanatosis (Fig. 2C). Registramos este comportamiento en dos ocasiones durante la observación y posterior manipulación del individuo.

Esta estrategia de defensa ha sido reportada como una respuesta ante posibles depredadores y se ha observado en otras especies de Bolitoglossa (B. flavimembris, B. franklini, B. morio, B. resplendens, B. rostrata, B. subpalmata), así como Nototriton picadoi, Oedipina poelzi y Oedipina uniformis (Dodd \& Brodie, 1976; Brodie, 1977). Las salamandras que se dejan caer y permanecen inmóviles pueden evadir a los depredadores porque pueden confundirse con su medio y hacer difícil su relocalización, reduciendo así el riesgo y la intensidad de un ataque (Brodie, 1974; Dodd \& Brodie, 1976).

Por su parte, el individuo de O. savagei al ser fotografiado con el flash activado tuvo movimientos de evasión y levantó su cola cerca de $90^{\circ}$ con relación a su cuerpo (Fig. 3 C). Este comportamiento ha sido reportado para Triturus marmoratus y Rhyacotriton olympicus, y especies de los géneros Taricha, Ambystoma y Eurycea, las cuales ante una amenaza pueden desplegar posiciones para confundir o atraer a los depredadores a partes del cuerpo menos comprometedoras (Brodie, 1977). Una de ellas consiste en levantar la cola para proteger los órganos 


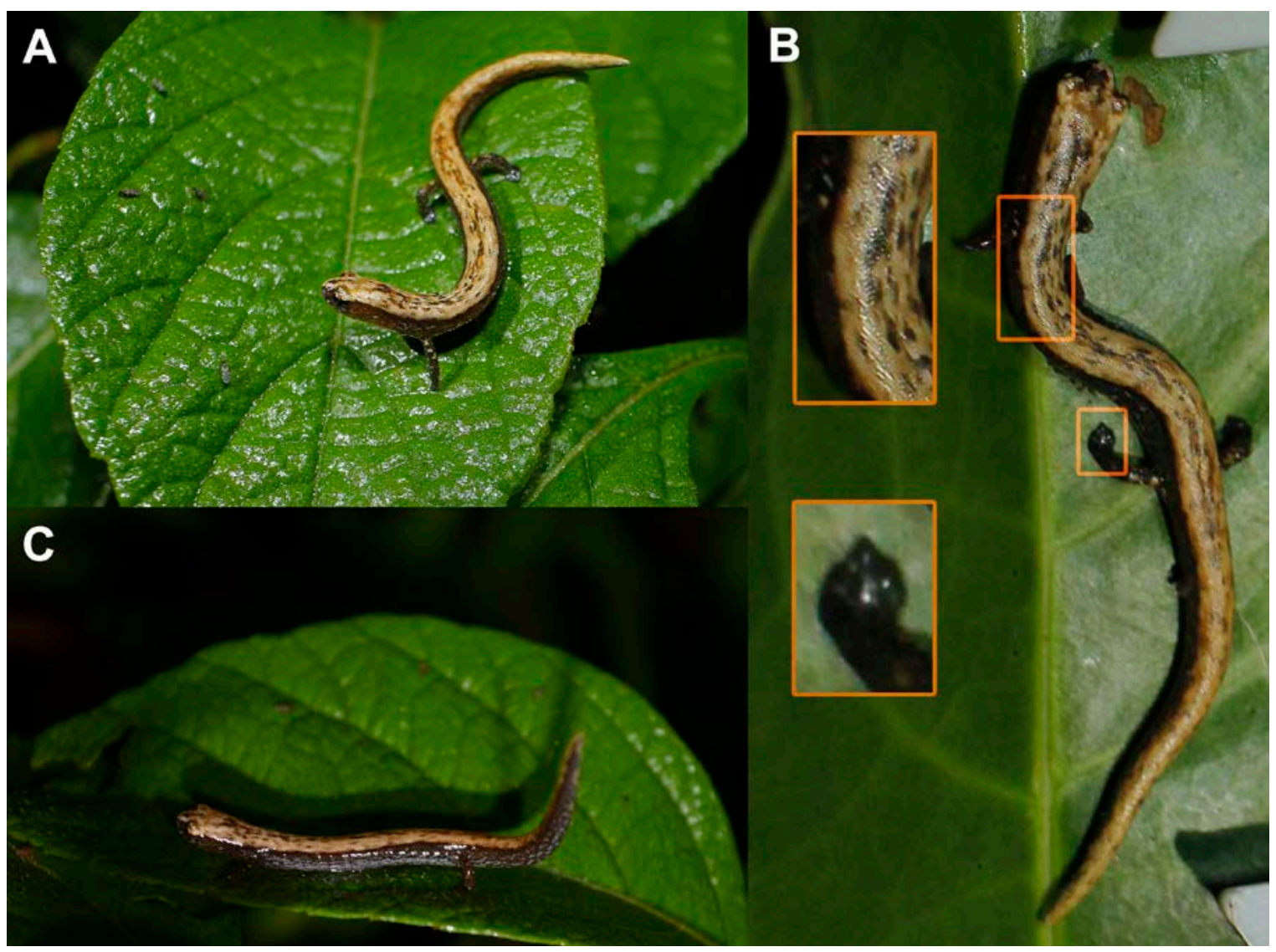

Figure 3. Oedipina savagei individual found in a forest-pasture ecotone at Las Tablas Protected Zone buffer area, Costa Rica (A). Characteristics used for identification: light dorsal stripe, longest toe is pointed (B), defense behavior holding tail elevated about $90^{\circ}$ angle with the body (C).

Figura 3. Individuo de Oedipina savagei encontrado en un ecotono de bosque-pastizal en el área de amortiguamiento de la Zona Protectora Las Tablas, Costa Rica (A). Características utilizadas para su identificación: raya dorsal clara, dedo más largo del pie terminado en punta (B); comportamiento de defensa levantando la cola cerca de $90^{\circ}$ con respecto a su cuerpo (C).

vitales, ya que estos anfibios realizan autotomía caudal y pueden poseer glándulas con toxinas nocivas en el dorso de la cola, como sucede en especies de los géneros Dicamptodon, Rhyacotriton, Ambystoma, Hynobius, Eurycea, Plethodon y Ensatina (Dodd \& Brodie, 1976; Brodie, 1977; Brodie, 1983).

Aunque los comportamientos defensivos en anfibios en general y en las salamandras en particular son frecuentes, los reportes en la literatura son escasos. Una proporción importante de especies de salamandras neotropicales no tienen una descripción de sus comportamientos defensivos, posiblemente por vacíos de conocimiento en aspectos de su biología e historia natural como ocurre también en anuros (Sánchez-Paniagua \& Abarca, 2016).
De hecho, estas observaciones fueron casuales y posiblemente sean una muestra restringida del repertorio comportamental aún desconocido de estas salamandras. Por lo tanto, animamos a los herpetólogos a reportar observaciones de comportamiento para empezar a llenar los vacíos de conocimiento de las salamandras, especialmente en áreas con alta diversidad taxonómica como es la región de Talamanca.

Agradecimientos.- Agradecemos a la finca Las Alturas del Bosque Verde y ProCAT Internacional, ya que las observaciones fueron realizadas durante proyectos dirigidos por estas. A Laia Gaitán por su apoyo en el trabajo de campo. A los revisores anónimos que nos ayudaron a mejorar notablemente el manuscrito. A Kristie Falconer por su revisión del resumen en inglés. 


\section{LITERATURA CITADA}

Bolaños, F. \& D.B. Wake. 2009. Two new species of montane webfooted salamanders (Plethodontidae: Bolitoglossa) from the Costa Rica-Panamá border region. Zootaxa 1981:57-68.

Boza-Oviedo, E., S.M. Rovito, G. Chaves, A. García-Rodríguez, L.G. Artavia, F. Bolaños, \& D.B. Wake. 2012. Salamanders from the eastern Cordillera de Talamanca, Costa Rica, with descriptions of five new species (Plethodontidae: Bolitoglossa, Nototriton, and Oedipina) and natural history notes from recent expeditions. Zootaxa 3309:36-61.

Brodie, E.D., J.A. Johnson \& C.K. Dodd. 1974. Immobility as a Defensive Behavior in Salamanders. Herpetologica 30:79-85.

Brodie, E.D. 1977. Salamander Antipredator Postures. Copeia 1977:523-535.

Brodie, E.D. 1983. Antipredator Adaptations of Salamanders: Evolution and Convergence Among Terrestrial Species. Pp. 109133. En N.S. Margaris, M. Arianoutsou-Faraggitaki, R.J. Reiter (Eds.), Adaptations to Terrestrial Environments. Plenum Press, New York, USA.

Brodie, E.D., P.K. Ducey \& E.A. Baness. 1991. Antipredator skin secretions of some tropical salamanders (Bolitoglossa) are Toxic to Snake Predators. Biotropica 23:58-62.

Dodd, C.K. \& E.D. Brodie. 1976. Defensive mechanisms of neotropical salamanders with an experimental analysis of immobility and the effect of temperature on immobility. Herpetologica 32:269-290.
Ducey P.K. \& E.D. Brodie. 1991. Evolution of antipredator behavior: individual and populational variation in a neotropical salamander. Herpetologica 47:89-95.

Ducey P.K. 1993. Salamander tail autotomy and snake predation: role of antipredator behavior and toxicity for three neotropical Bolitoglossa (Caudata: Plethodontidae). Biotropica 25:344-349.

García-París, M. \& D.B. Wake. 2000. Molecular phylogenetic analysis of relationships of the tropical salamander genera Oedipina and Nototriton, with descriptions of a new genus and three new species. Copeia 2000:42-70.

Kubicki, B. \& E. Arias. 2016. A beautiful new yellow salamander, genus Bolitoglossa (Caudata: Plethodontidae), from the northeastern slopes of the Cordillera de Talamanca, Costa Rica. Zootaxa 4184:329-346.

Sánchez-Paniagua, K. \& J.G. Abarca. 2016. Thanatosis in four poorly known toads of the genus Incilius (Amphibia: Anura) from the highlands of Costa Rica. Mesoamerican Herpetology 3:135-140.

Vial, J.L. 1968. The ecology of the tropical salamander, Bolitoglossa subpalmata, in Costa Rica. Revista de Biología Tropical 15:13-115.

Wake, D.B., J.M. Savage \& J. Hanken. 2007. Montane salamanders from the Costa Rica-Panamá border region, with descriptions of two new species of Bolitoglossa. Copeia 2007:556-565.

Zumbado-Ulate, H., K.N. Nelson, A. García-Rodríguez, G. Chaves, E. Arias, F. Bolaños, S.M. Whitfield \& C.L. Searle. 2019. Endemic infection of Batrachochytrium dendrobatidis in Costa Rica: Implications for amphibian conservation at regional and species level. Diversity 11:129. 